\title{
Role of estrogen receptor in breast cancer cell gene expression
}

\author{
YABING ZHENG $^{1}$, XIYING SHAO $^{1}$, YUAN HUANG $^{1}$, LEI SHI $^{1}$, BO CHEN $^{2}$, XIAOJIA WANG $^{1}$, \\ HONGJIAN YANG ${ }^{3},{\text { ZHANHONG } \text { CHEN }^{1} \text { and XIPING ZHANG }}^{3}$ \\ Departments of ${ }^{1}$ Medical Oncology (Breast), ${ }^{2}$ Pathology and ${ }^{3}$ Breast Surgery, \\ Zhejiang Cancer Hospital, Hangzhou, Zhejiang 310022, P.R. China \\ Received April 28, 2015; Accepted February 23, 2016
}

DOI: $10.3892 / \mathrm{mmr} .2016 .5018$

\begin{abstract}
The aim of the present study was to establish the underlying regulatory mechanism of estrogen receptor (ER) in breast cancer cell gene expression. A gene expression profile (accession no. GSE11324) was downloaded from the Gene Expression Omnibus (GEO) database. Differentially expressed genes (DEGs) from an estrogen treatment group and a control group were identified. Chromatin immunoprecipitation with high-throughput sequencing data (series GSE25710) was obtained from the GEO for the ER binding sites, and binding and expression target analysis was performed. A total of 3,122 DEGs were obtained and ER was demonstrated to exhibit inhibition and activation roles during the regulation of its target gene expression. Motif analysis revealed that the upregulated target genes that demonstrated interactions with ER were meis homeobox 1 (MEIS1) and forkhead box P3 (FOXP3). The downregulated target genes, which demonstrated interactions with ER, were thyroid hormone receptor, $\beta$ (THRB) and grainyhead-like 1 (GRHL1). Thus, it was observed that ER stimulated gene expression by interacting with MEISI and $F O X P 3$, and ER inhibited gene expression by interacting with THRB and GRHL1. However, additional experiments are required to provide further confirmation of these findings.
\end{abstract}

\section{Introduction}

Breast cancer is a common types of malignancy among females, worldwide (1). Genetic and epigenetic alterations are involved in the underlying mechanisms associated with breast cancer development (2-4). Although therapeutic and diagnostic methods have improved, this type of cancer remains the primary cause of cancer-associated mortality among females (5). It is estimated that there are 464,000 cases of breast cancer, accounting for $13.5 \%$ of all cancer cases in

Correspondence to: Dr Xiying Shao, Department of Medical Oncology (Breast), Zhejiang Cancer Hospital, 38 Guangji Road, Banshan, Hangzhou, Zhejiang 310022, P.R. China

E-mail: xiyingsh@163.com

Key words: estrogen receptor, breast cancer, differentially expressed gene
Europe in 2012, and the number of breast cancer-associated mortalities is 131,000 (6). Furthermore, breast cancer is the most common cause of cancer-associated mortality in females. Therefore, it is essential to understand its molecular mechanism and develop more effective therapeutic methods for breast cancer treatment.

The estrogen receptor (ER) is critical in determining the phenotype of human breast cancers and is one of the most important therapeutic targets (7). Furthermore, certain studies have suggested that activation of ER is responsible for various biological processes, including cell growth and differentiation, and programmed cell death $(8,9)$. It is reported that the response of ER to estrogen is critical in controlling specific protein synthesis (10). ER-mediated transcription has been extensively investigated on a small number of endogenous target promoters $(11,12)$. Carroll et al (13) identified various novel features of ER transcription, including an involvement of distal cis-regulatory enhancer regions, and a requirement for the Forkhead protein, FoxA1, in facilitating ER binding to chromatin and subsequent gene transcription. However, the mechanisms underlying estrogen-associated gene expression changes in breast cancer remain poorly understood.

The human cancer cell line, MCF7, contains ER and demonstrates an estrogen response (14). In the present study, the microarray data was obtained from the Gene Expression Omnibus (GEO) database, which was developed using MCF7 cells stimulated with estrogen for different durations. The differentially expressed genes (DEGs) between the control and estrogen treatment groups were analyzed. The ER binding sites were identified and motif analysis was performed. The aim was to investigate the regulatory mechanism of ER in the progression of breast cancer.

\section{Materials and methods}

Affymetrix microarray data. The mRNA microarray datasets (accession no. GSE11324) were obtained from the GEO database (http://www.ncbi.nlm.nih.gov/geo/), which was deposited by Carroll et al (13). The gene expression profiles were developed from 12 batches of MCF7 cells that had been treated with $100 \mathrm{nM}$ estrogen for $0,3,6$ or $12 \mathrm{~h}$. The experiment had been repeated three times.

Data preprocessing and DEG Identification. The raw Affymetrix CEL data were downloaded based on the platform of 


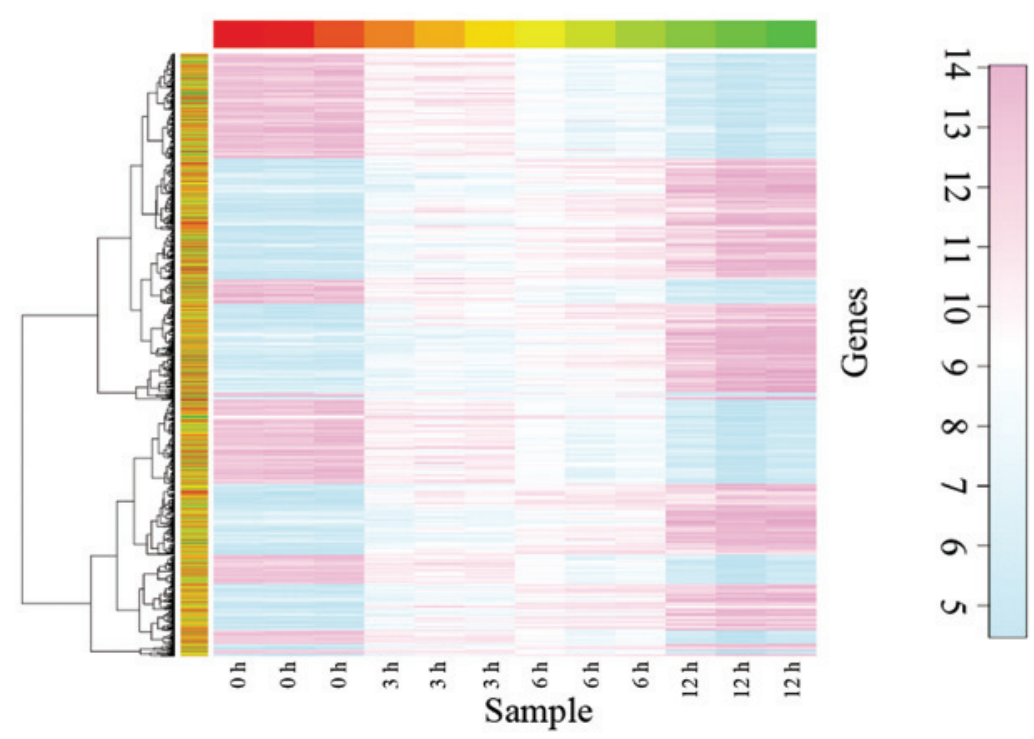

Figure 1. Heatmap showing the results of hierarchical cluster analysis of DEGs at four different time-points. The gene expression levels from high to low are indicated in red to green, respectively, and the number of DEGs is expressed in pink to blue as indicated. DEG, differentially expressed gene.

GPL570 (HG-U133_Plus_2) Affymetrix GeneChip ${ }^{\circledR}$ Human Genome U133 Plus 2.0 Array (Affymetrix, Santa Clara, CA, USA). To obtain the expression matrix, mRNA expression data were first preprocessed using the Affy Package in R language (http://bioconductor.org/packages/release/bioc/html/affy.

html) (15). When multiple probes mapped to the same Entrez Gene ID, the mean expression value of these probes was calculated for the gene.

The DEGs between the ER-stimulated MCF7 cells were screened by limma package in R language (16) and genes with an adjusted P-value $<0.05$ were considered to be DEGs. Subsequently, the DEGs in the 3 vs. $0 \mathrm{~h}, 6$ vs. $0 \mathrm{~h}$ and $12 \mathrm{vs.} 0 \mathrm{~h}$ groups underwent hierarchical clustering analysis (17) using the pheatmap package in $\mathrm{R}$ language (R Core Team, Vienna, Austria).

ER binding site analysis. Chromatin immunoprecipitation with high-throughput sequencing (ChIP-seq) data was obtained from the GEO repository (accession no. GSE25710; ChIP-seq for forkhead box A1, ER and CCCTC-binding factor in breast cancer cell lines). The ChIP-seq data were treated with ER antibody (Ab-10; Neomarkers, Lab Vision; Fremont, CA, USA) and mapped to the whole genome sequence using BowTie software version 2.1.0 (https://sourceforge.net/projects/bowtie-bio/files/bowtie2/) (18). To identify the possible ER binding sites, peak calling was performed by model-based analysis of ChIP-Seq (MACS; version 1.4.2) (19). The q-value was set at $<0.01$ and served as the cut-off to improve the ChIP-seq peak detection. The DNA sequence fragments that interacted with ER in different regions of the whole genome were evaluated using the Cis-regulatory Element Annotation System software (http://liulab.dfci.harvard.edu/CEAS) and a graph of the results was constructed $(20,21)$.

Binding and expression target analysis (BETA). BETA version 1.0.7 (http://cistrome.org/BETA/) (22) is a software package that predicts target genes through the analysis of ChIP-seq data and DEGs. The ER target genes were screened, using BETA software, based on the ER-associated ChIP-seq data and ER-stimulated DEG data. Briefly, the genes within a $100-\mathrm{kb}$ distance of the significant top 10,000 peaks were collected. These genes were ranked based on their distance from the peak and the significance of their differential expression. The genes ranked at the top exhibited the greatest possibility of being regulated by ER.

Subsequently, the regulatory function (activation or inhibition) of ER among the top 500 DEGs in the 12 vs. 0 h group, which neared the top 10,000 peaks were evaluated by BETA analysis. The motifs of the ER binding sites were obtained and the factors that interacted with ER were predicted (23).

\section{Results}

Identification of DEGs. The DEGs with adjusted $\mathrm{P}<0.05$ in the 3 vs. $0 \mathrm{~h}, 6$ vs. $0 \mathrm{~h}$ and 12 vs. $0 \mathrm{~h}$ groups were screened out. Subsequently, the gene expression profiles of DEGs at different time-points were analyzed. Hierarchical clustering indicated that the DEGs were clearly separated and the difference in gene expression became more pronounced with increasing duration of estrogen treatment (Fig. 1). In order to obtain more reliable results, a total of 3,122 DEGs with adjusted $\mathrm{P}<0.01$ in the 12 vs. $0 \mathrm{~h}$ group were selected for further analysis, including 1,755 upregulated and 1,366 downregulated genes.

Identification of ER-specific binding sites. Based on the ChIP-seq peaks determined by MACS and the cut-off value of $\mathrm{q}<0.01$, a total of 10,058 peaks were obtained. Based on the ChIP-seq data analysis, the distribution of ER binding sites in the whole genome was determined. Fig. 2 demonstrates that the DNA sequence fragments that interact with the ER are located in different regions of the whole genome, such as promoter, downstream and intergenic regions.

BETA analysis. In order to analyze the regulatory effects of ER on its target genes, the 10,000 most significant peaks and 
A

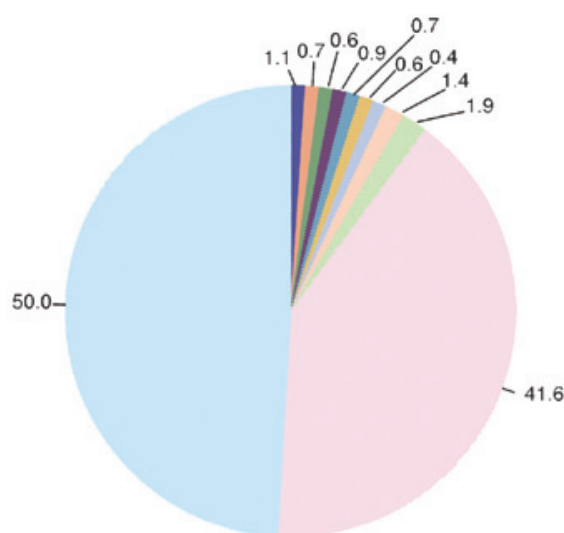

- Promoter ( $\leq 1000 \mathrm{bp}): 1.1 \%$

" Promoter (1000-2000 bp): 0.7\%

- Promoter (2000-3000 bp): 0.6\%

- Downstream ( $\leq 1000 \mathrm{bp}$ ): $0.9 \%$

- Downstream (1000-2000 bp): 0.7\%

= Downstream (2000-3000 bp): $0.6 \%$

5'UTR: $0.4 \%$

3'UTR: $1.4 \%$

Coding exon: $1.9 \%$

Intron: $41.6 \%$

Distal intergenic: $50.0 \%$
B

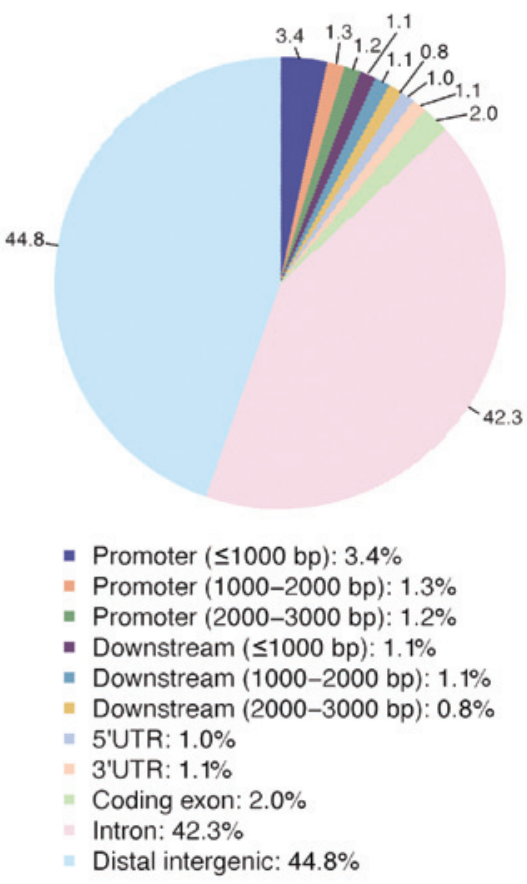

Figure 2. Distribution of ER binding sites in the genome. (A) Prediction of ER binding sites in the whole genome. (B) ER binding sites tested by chromatin immunoprecipitation sequencing, indicating a higher number of ER binding sites in the promoter region compared with A. ER, estrogen receptor; UTR, untranslated region.

the 500 most significant DEGs in the 12 vs. 0 h group were selected for BETA analysis. Results of the BETA analysis indicated that the ER exerts an inhibitory role in addition to an activating role regarding the regulation of its target genes (Fig. 3). In addition, motif analysis revealed that ER may exert an activation role in gene expression by interacting with MEIS1 and FOXP3 (Table I, Part A) and inhibit gene expression by interacting with THRB and GRHL1 (Table I, Part B).

\section{Discussion}

The ER is recognized as the master transcriptional regulator of the breast cancer phenotype and is critical in predicting the early recurrence of breast cancer. However, to the best of our knowledge, the role of ER in breast cancer cell gene expression has not been clearly clarified. In the present study, DEGs in the MCF7 breast cancer cell line that had been stimulated by estrogen for different durations were analyzed. Using a combination of the ChIP-seq dataset and the identified DEGs, the ER target and response genes were predicted. A set of cis-acting targets across the whole genome of the ER were identified.

The present results demonstrated that 3,122 genes were differentially expressed as a result of estrogen stimulation. The hierarchical clustering analysis for the DEGs indicated that the long-term stimulation by estrogen improved the differential gene expression in breast cancer cells. Using motif analysis, ER was identified to inhibit and stimulate target gene expression, which was demonstrated by interactions between MEIS1, FOXP3, THRB and GRHL1, and ER.

In the present study, ER was found to activate gene expression by interacting with MEIS1 and FOXP3. MEISI

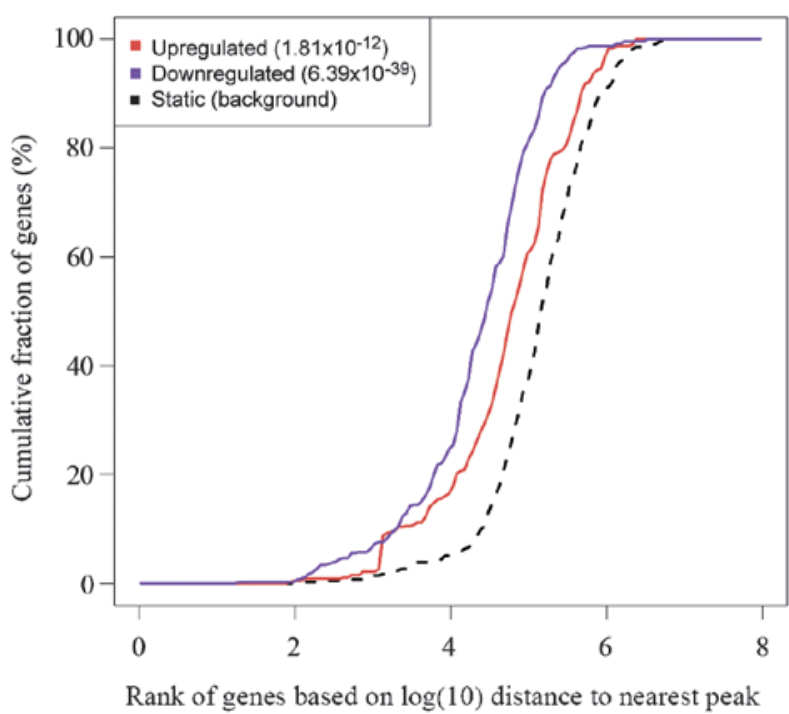

Figure 3. ER activating/repressive function prediction. Genes were cumulated by ranking on the basis of the regulatory potential score from high to low. A significance of ER activation/inhibition in gene expression was indicated. ER, estrogen receptor.

is a homeobox gene, and encodes the homeobox protein, MEIS1 (24). In addition, certain homeobox proteins are associated with tumor formation; Mahmoud et al (25) proposed MEIS1 as a critical transcriptional regulator of cardiomyocyte proliferation and as a potential therapeutic target for heart regeneration. It was previously reported that MEIS1 is a prognostic and predictive biomarker for breast cancer (26) and a recent study indicated that MEIS1, as a HOX gene, is associated with decreased proliferation in the mesenchymal 
Table I. Motifs in the target genes.

A, Upregulated target genes

\begin{tabular}{lllr}
\hline Symbol & \multicolumn{1}{c}{ DNA-binding domain } & Species & P-value $(t$-test $)$ \\
\hline ESR1 & Nuclear hormone receptor family & Homo sapiens & $2.24 \times 10^{-40}$ \\
ESRRB & Nuclear hormone receptor family & Homo sapiens & $2.24 \times 10^{-40}$ \\
ESRRA & Nuclear hormone receptor family & Homo sapiens & $2.24 \times 10^{-40}$ \\
ESRRG & Nuclear hormone receptor family & Homo sapiens & $2.24 \times 10^{-40}$ \\
RARA & Nuclear hormone receptor family & Homo sapiens & $2.24 \times 10^{-40}$ \\
PPARG & Nuclear hormone receptor family & Homo sapiens & $2.24 \times 10^{-40}$ \\
NR2F1 & Nuclear hormone receptor family & Homo sapiens & $2.24 \times 10^{-40}$ \\
ESR2 & Nuclear hormone receptor family & Homo sapiens & $2.24 \times 10^{-40}$ \\
MEIS1 & Homeo domain family & Homo sapiens & $1.90 \times 10^{-18}$ \\
FOXP3 & Forkhead domain family & Homo sapiens & 14.50 \\
& & & $2.52 \times 10^{-9}$ \\
\hline
\end{tabular}

B, Downregulated target genes

\begin{tabular}{lllr}
\hline Symbol & \multicolumn{1}{c}{ DNA-binding domain } & Species & P-value $(t$-test) \\
\hline ESR1 & Nuclear hormone receptor family & Homo sapiens & $1.12 \times 10^{-43}$ \\
ESRRB & Nuclear hormone receptor family & Homo sapiens & $1.12 \times 10^{-43}$ \\
ESRRA & Nuclear hormone receptor family & Homo sapiens & $1.12 \times 10^{-43}$ \\
ESRRG & Nuclear hormone receptor family & Homo sapiens & $1.12 \times 10^{-43}$ \\
RARA & Nuclear hormone receptor family & Homo sapiens & $1.12 \times 10^{-43}$ \\
PPARG & Nuclear hormone receptor family & Homo sapiens & $1.12 \times 10^{-43}$ \\
NR2F1 & Nuclear hormone receptor family & Homo sapiens & $1.12 \times 10^{-43}$ \\
ESR2 & Nuclear hormone receptor family & Homo sapiens & $1.12 \times 10^{-43}$ \\
THRB & Nuclear hormone receptor family & Homo sapiens & $7.83 \times 10^{-12}$ \\
GRHL1 & CP2 transcription factor domain family & Homo sapiens & 14.58 \\
\hline
\end{tabular}

ESR1, estrogen receptor 1; ESRRB, estrogen related receptor $\beta$; ESRRA, estrogen related receptor $\alpha$; ESRRG, estrogen related receptor $\gamma$; RARA, retinoic acid receptor $\alpha$; PPARG, peroxisome proliferator activated receptor $\gamma$; NR2F1, nuclear receptor subfamily 2 group F member 1 ; ESR2, estrogen receptor 2 (ER $\beta$ ); MEIS1, meis homeobox 1; FOXP3, forkhead box P3; THRB, thyroid hormone receptor, $\beta$; GRHL1, grainyhead-like 1 .

stem-like subtype of breast cancer (27). Furthermore, the androgen/estrogen metabolism pathway is responsible for ER-negative breast cancer (27). Therefore, whether the MEIS1 response to ER is responsible for the ER-positive breast cancer subtype requires further investigation.

FOXP3, a member of the FOX protein family, is involved in the immune system response. FOXP3 controls the expression of numerous genes and has recently been reported to be expressed in tumor cells (28). FOXP3 expression was reported to be enhanced in estrogen-treated mice (29). Fox et al (30) showed that expression of the transcription factor, FOXP1 is associated with ER $\alpha$ and improved survival in patients with primary breast carcinomas. Merlo et al (28) suggested that FOXP3 expression was a novel independent prognostic factor for breast carcinoma. Thus, ER may interact with MEISI and $F O X P 3$ to activate gene expression in breast cancer. In addition, the results of the present study showed that ER suppressed gene expression via THRB and GRHL1. THRB is considered to be a potential cancer suppressor (31) and $T H R B$ gene silencing by aberrant methylation is highly prevalent in breast cancer patients (31). Furthermore, the loss of THRB expression as a result of methylation may be a plasma biomarker for the prognosis of breast cancer patients (31). Baniahmad et al (32) identified that the interaction of THRB with transcription factors may mediate the activation of the target gene. GRHL1 inhibits tumorigenicity and is a prognostic marker in neuroblastoma $(33,34)$. Tao et al $(35)$ found that Xenopus GRHL1 is essential for epidermal differentiation. de la Garza et al (36) indicated that interferon regulatory factor 6 promoted differentiation of the periderm by stimulating the expression of grainyhead-like 3 (36). Although the evidence for the interaction between ER and THRB and GRHL1 is insufficient, THRB and GRHL may be potential targets to analyze the function of ER in breast cancer.

In conclusion, ER may activate or suppress gene expression by interacting with MEISI and FOXP3, or THRB and GRHL1, respectively. These data may be useful for identifying novel therapeutic agents and designing clinical trials; however, further experiments are required to confirm the results. 


\section{References}

1. Siegel R, Ma J, Zou Z and Jemal A: Cancer statistics, 2014. CA Cancer J Clin 64: 9-29, 2014.

2. Dworkin AM, Huang TH and Toland AE: Epigenetic alterations in the breast: Implications for breast cancer detection, prognosis and treatment. Semin Cancer Biol 19: 165-171, 2009.

3. Jovanovic J, Rønneberg JA, Tost J and Kristensen V: The epigenetics of breast cancer. Mol Oncol 4: 242-254, 2010.

4. Polyak K: Breast cancer: Origins and evolution. J Clin Invest 117: 3155-3163, 2007.

5. Jemal A, Bray F, Center MM, Ferlay J, Ward E and Forman D: Global cancer statistics. CA Cancer J Clin 61: 69-90, 2011.

6. Ferlay J, Steliarova-Foucher E, Lortet-Tieulent J, Rosso S, Coebergh JW, Comber H, Forman D and Bray F: Cancer incidence and mortality patterns in Europe: Estimates for 40 countries in 2012. Eur J Cancer 49: 1374-1403, 2013.

7. Ding LH, Ye QN, Lu QJ, Zhu JH, Yan JH, Wang ZH and Huang CF: Expression of XBP-1 in breast cancer cell lines and its role in ERalpha signaling. Yi Chuan Xue Bao 31: 380-384, 2004 (In Chinese)

8. Katzenellenbogen BS: Estrogen receptors: Bioactivities and interactions with cell signaling pathways. Biol Reprod 54: 287-293, 1996.

9. Katzenellenbogen BS, Montano MM, Ekena K, Herman ME and McInerney EM: William L. McGuire Memorial Lecture. Antiestrogens: Mechanisms of action and resistance in breast cancer. Breast Cancer Res Treat 44: 23-38, 1997.

10. Horwitz KB and McGuire WL: Estrogen control of progesterone receptor in human breast cancer: Correlation with nuclear processing of estrogen receptor. J Biol Chem 253: 2223-2228, 1978.

11. Shang Y, Hu X, DiRenzo J, Lazar MA and Brown M: Cofactor dynamics and sufficiency in estrogen receptor-regulated transcription. Cell 103: 843-852, 2000

12. Shang Y and Brown M: Molecular determinants for the tissue specificity of SERMs. Science 295: 2465-2468, 2002.

13. Carroll JS, Meyer CA, Song J, Li W, Geistlinger TR, Eeckhoute J, Brodsky AS, Keeton EK, Fertuck KC, Hall GF, et al: Genome-wide analysis of estrogen receptor binding sites. Nat Genet 38: 1289-1297, 2006.

14. Lippman M, Bolan G and Huff K: The effects of estrogens and antiestrogens on hormone-responsive human breast cancer in long-term tissue culture. Cancer Res 36: 4595-4601, 1976.

15. Irizarry R, Hobbs B, Collin F, Beazer-Barclay YD, Antonellis KJ, Scherf U and Speed TP: Exploration, normalization and summaries of high density oligonucleotide array probe level data. Biostatistics 4: 249-264, 2003.

16. Smyth GK: limma: Linear models for microarray data. In: Bioinformatics and Computational Biology Solutions Using R and Bioconductor. Gentleman R, Carey VJ, Huber W, Irizarry RA and Dudoit S (eds). 1st edition. Springer New York, NY, USA pp397-420, 2005

17. Langfelder $P$ and Horvath $S$ : Fast R functions for robust correlations and hierarchical clustering. J Stat Softw 46: pii: i11, 2012.

18. Langmead B, Trapnell C, Pop M and Salzberg SL: Ultrafast and memory-efficient alignment of short DNA sequences to the human genome. Genome Biol 10: R25, 2009

19. Feng J, Liu T and Zhang Y: Using MACS to identify peaks from ChIP-Seq data. Curr Protoc Bioinformatics Chapter 2: Unit 2. 14, 2011.

20. Zhang Y,Liu T, Meyer CA,Eeckhoute J, Johnson DS, Bernstein BE, Nusbaum C, Myers RM, Brown M, Li W and Liu XS: Model-based analysis of ChIP-Seq (MACS). Genome Biol 9: R137, 2008.
21. Shin H, Liu T, Manrai AK and Liu XS: CEAS: Cis-regulatory element annotation system. Bioinformatics 25: 2605-2606, 2009.

22. Grober OM, Mutarelli M, Giurato G, Ravo M, Cicatiello L, De Filippo MR, Ferraro L, Nassa G, Papa MF, Paris O, et al: Global analysis of estrogen receptor beta binding to breast cancer cell genome reveals an extensive interplay with estrogen receptor alpha for target gene regulation. BMC Genomics 12: 36, 2011.

23. Wang S, Sun H, Ma J, Zang C, Wang C, Wang J, Tang Q, Meyer CA, Zhang Y and Liu XS: Target analysis by integration of transcriptome and ChIP-seq data with BETA. Nat Protoc 8: 2502-2515, 2013

24. Moskow JJ, Bullrich F, Huebner K, Daar IO and Buchberg AM: Meis1, a PBX1-related homeobox gene involved in myeloid leukemia in BXH-2 mice. Mol Cell Biol 15: 5434-5443, 1995.

25. Mahmoud AI, Kocabas F, Muralidhar SA, Kimura W, Koura AS Thet S, Porrello ER and Sadek HA: Meis1 regulates postnatal cardiomyocyte cell cycle arrest. Nature 497: 249-253, 2013.

26. Doolan P, Clynes M, Kennedy S, Mehta JP, Germano S Ehrhardt C, Crown J and O'Driscoll L: TMEM25, REPS2 and Meis 1: Favourable prognostic and predictive biomarkers for breast cancer. Tumour Biol 30: 200-209, 2009.

27. Lehmann BD, Bauer JA, Chen X, Sanders ME, Chakravarthy AB, Shyr Y and Pietenpol JA: Identification of human triple-negative breast cancer subtypes and preclinical models for selection of targeted therapies. J Clin Invest 121: 2750-2767, 2011.

28. Merlo A, Casalini P, Carcangiu ML, Malventano C, Triulzi T, Mènard S, Tagliabue E and Balsari A: FOXP3 expression and overall survival in breast cancer. J Clin Oncol 27: 1746-1752, 2009.

29. Polanczyk MJ, Hopke C, Huan J, Vandenbark AA and Offner H: Enhanced FoxP3 expression and Treg cell function in pregnant and estrogen-treated mice. J Neuroimmunol 170: 85-92, 2005.

30. Fox SB, Brown P, Han C, Ashe S, Leek RD, Harris AL and Banham AH: Expression of the forkhead transcription factor FOXP1 is associated with estrogen receptor alpha and improved survival in primary human breast carcinomas. Clin Cancer Res 10: 3521-3527, 2004.

31. Ling Y, Xu X, Hao J, Ling X, Du X, Liu X and Zhao X: Aberrant methylation of the THRB gene in tissue and plasma of breast cancer patients. Cancer Genet Cytogenet 196: 140-145, 2010.

32. Baniahmad A, Ha I, Reinberg D, Tsai S, Tsai MJ and O'Malley BW: Interaction of human thyroid hormone receptor beta with transcription factor TFIIB may mediate target gene derepression and activation by thyroid hormone. Proc Natl Acad Sci USA 90: 8832-8836, 1993.

33. Fabian J, Lodrini M, Schier M, Thole T, Kopp-Schneider A, Capper D, von Deimling A, Oehme I, Wiegand I, Milde T, et al: GRHL1 inhibits tumorigenicity and is a prognostic marker in neuroblastoma. Klin Padiatr 225: A29, 2013.

34. Fabian J, Lodrini M, Oehme I, Schier MC, Thole TM, Hielscher T, Kopp-Schneider A, Opitz L, Capper D, von Deimling A, et al: GRHL1 acts as tumor suppressor in neuroblastoma and is negatively regulated by MYCN and HDAC3. Cancer Res 74: 2604-2616, 2014.

35. Tao J, Kuliyev E, Wang X, Li X, Wilanowski T, Jane SM, Mead PE and Cunningham JM: BMP4-dependent expression of Xenopus Grainyhead-like 1 is essential for epidermal differentiation. Development 132: 1021-1034, 2005.

36. de la Garza G, Schleiffarth JR, Dunnwald M, Mankad A, Weirather JL, Bonde G, Butcher S, Mansour TA, Kousa YA, Fukazawa $\mathrm{CF}$, et al: Interferon regulatory factor 6 promotes differentiation of the periderm by activating expression of grainyhead-like 3. J Invest Dermatol 133: 68-77, 2013. 